\title{
Erratum to: Vertical Profiles of Community Abundance and Diversity of Anaerobic Methanotrophic Archaea (ANME) and Bacteria in a Simple Waste Landfill in North China
}

\author{
Jun Dong ${ }^{1}$ - Linjie Ding ${ }^{1} \cdot$ Xu Wang ${ }^{1} \cdot$ Zifang Chi $^{1}$ • \\ Jiansen Lei ${ }^{1}$
}

(C) Springer Science+Business Media New York 2015

Erratum to: Appl Biochem Biotechnol (2015) 175:2729-2740

DOI 10.1007/s12010-014-1456-3

The original version of this article unfortunately contained an error.

The primary address for corresponding author Zifang Chi (Z. Chi) was listed incorrectly as Key Laboratory for Solid Waste Management and Environment Safety, Ministry of Education, Tsinghua University, Beijing 100084, People's Republic of China.

Dr. Chi's correct primary address should be Key Lab of Groundwater Resources and Environment, Ministry of Education, Jilin University, Changchun 130021, People's Republic of China.

The online version of the original article can be found at http://dx.doi.org10.1007/s12010-014-1456-3.

Zifang Chi

chizifang@jlu.edu.cn

1 Key Lab of Groundwater Resources and Environment, Ministry of Education, Jilin University, Changchun 130021, People's Republic of China 